\title{
Mobile Computing Application for Industrial Field Service Engineering: A Case for ABB Service Engineers
}

\author{
Welderufael B. Tesfay ${ }^{1}$, Markus Aleksy ${ }^{2}$, Karl Andersson ${ }^{1}$, and Marko Lehtola ${ }^{3}$ \\ ${ }^{1}$ Pervasive and Mobile Computing Laboratory \\ Luleå University of Technology \\ SE-971 87 Luleå, Sweden \\ \{weltes-0@student., karl.andersson@\}1tu.se \\ ${ }^{2}$ ABB Corporate Research \\ Industrial Software Systems Program \\ Ladenburg, Germany \\ markus.aleksy@de.abb.com \\ ${ }^{3}$ ABBCorporate Research \\ Industrial Software Systems Program \\ Västerås, Sweden \\ marko.lehtola@se.abb.com
}

\begin{abstract}
The increasing digitalization of our daily lives is gaining a new momentum recently in what is called the postdesktop era of computing. This wide spread in digitalization is made possible by the introduction of handy devices specifically of mobile phones, laptop computers, Smartphones, and tablets. This new trend in shaping up technological advancement presents new opportunities for mobile information presentation, processing, and synthesis in different spheres of application such as providing industrial field services engineering support. Industrial field service engineers execute sets of work orders on daily basis plans. These work order executions demand the availability of digital information anywhere anytime to process them faster. This paper presents a scenario where the newly emerging Smartphone features such as augmented reality, audio / video processing, and near field communication sensing can be used to develop a prototype that supports industrial field service engineers in the contemporary field service engineering taskforces. It employs real world use-cases and carrying out quantitative prototype usability test analysis.
\end{abstract}

Index Terms - Mobile computing, Industrial field service, Android, Work orders, Augmented reality, Near field communication.

\section{INTRODUCTION}

In the vision of pervasive or ubiquitous computing set forward by Mark Weiser [11] information will be everywhere anytime, the physical and digital worlds interconnect, technology becomes invisible in our lives and a great number of devices and software components collaborate unobtrusively in a smart space to provide services to users. Notwithstanding the availability of the required technology, truly pervasive computing environments have not yet been realized; only prototypes and theoretical designs have been developed by the research community. Smartphones might well be the most powerful pervasive embedded device and the ideal platform to be used for input for pervasive computing [12]. With the advent of wireless networking, cellular telephone, packet radio, radio frequency identifiers (RFID), smart personal object technology (SPOT), global positioning systems (GPS), and wireless sensor networks mobile users are able to query their environment and they allow applications to monitor and track remote objects [16][17].

Industrial field service can greatly benefit from the advances in information and communication technology (ICT) in increasing the speed of information flow, enhancing the efficiency and effectiveness of information communication, and reducing the cost of information transfer. In this essence, mobile computing applications promise to be of great utilization interest in the industrial field service to pervasively avail on-site information especially improved process support by providing tailored service information and presenting it in an ad-hoc manner to service engineers [1].

Industrial field service engineers execute sets of work orders on daily basis plans. These work order executions demand the availability of digital information anywhere anytime to minimize the average time required to accomplish them. In this context, the pervasiveness and ubiquity of smart mobile devices creates a favorable possibility for industrial field service engineering automation. Most contemporary Smartphone devices come out with abundant features to use for industrial field service engineering task automations and different ways of presenting information to service engineers.

The project started with the hypothesis that the newly emerging Smartphone features functional combination can help mitigate the information availability deficit in industrial field services. The objective includes designing a prototype showcasing the possibility to use Smartphone features in industrial field service and carrying out prototype usability experiments. The prototype developed as proof of concept in this project enables service engineers to locate service fields, locate equipment, record work order events and report them for later use. 
The remainder of the paper is organized as follows: Section I discusses related work and section II focuses on the proposed solution architecture, utilized technologies, and prototype implementations whereas section III emphasizes on the experiments and evaluation followed by section IV on results. It then concludes on section $\mathrm{V}$ by highlighting the conclusions and future works.

\section{RELATED WORK}

In this section, we will discuss some works attempted to employ mobile computing technologies into industrial engineering. To our knowledge, there are not much projects / prototypes carried out in the same or similar scenario of collection of emerging Smartphone technologies as this project.

The contemporary industrial field service engineering faces the challenges of changing business environments, technological progress, and an aging workforce. Aleksy et al. [1], further discussed that these multidimensional challenges are more prevalent in high wage countries which bids for alternative solutions using current technologies.

The ubiquity of Smartphone devices with built-in cameras, accelerometer and magnetometers provides a good opportunity to do augmented reality (AR) applications. On the other hand, the limited processing power, small memory access, small cache and less performance of doing real-time image processing hinders it [8]. Another challenge is that most current systems require heavy and bulky head-mounted displays (HMDs) and are based on inflexible centralized architectures for detecting service locations and superimposing AR images [9].

AR has been researched and used in different application areas for almost two decades [10].

In their AR Computing Arena for Digital Entertainment (ARCADE) AR environment system, Lyu et al. [2] showed how the technology can be used for entertainment application development. ARCADE is a general AR platform that contains software tools, algorithms, designs, and configurations for entertainment applications [2].

It is estimated that NFC will be one of the widely deployed pervasive communication technology objects that will enable human beings to communicate with physical objects in their vicinity [13]. It is a new technology but it is maturing fast and finding many application areas especially by coupling it with Smartphone devices as it is being standardized [14]. Uckelmann et al. [15] suggest that NFC is the next hoped communication enabler to realize the Internet of Things (IoT). According to them, it has high potential for people to seamlessly identify things to access as well as contribute related information. Even though it is questionable how many mobile phone owners will use this utility technology, NFC is expected to be the next logical step for user interaction with the IoT [15].

\section{PROPOSED SOLUTION}

\section{A. Proposed Architectures}

The general architecture of the developed prototype corresponds to a 3-tier architecture that consists of a graphical

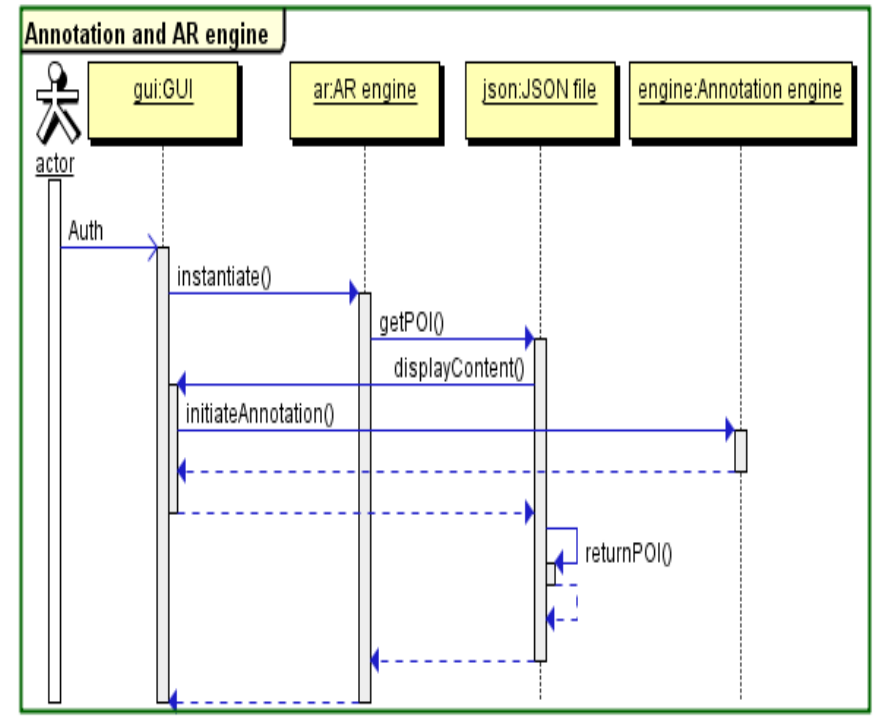

Figure 1: Sequence diagram of the annotation and AR engine modules of the prototype

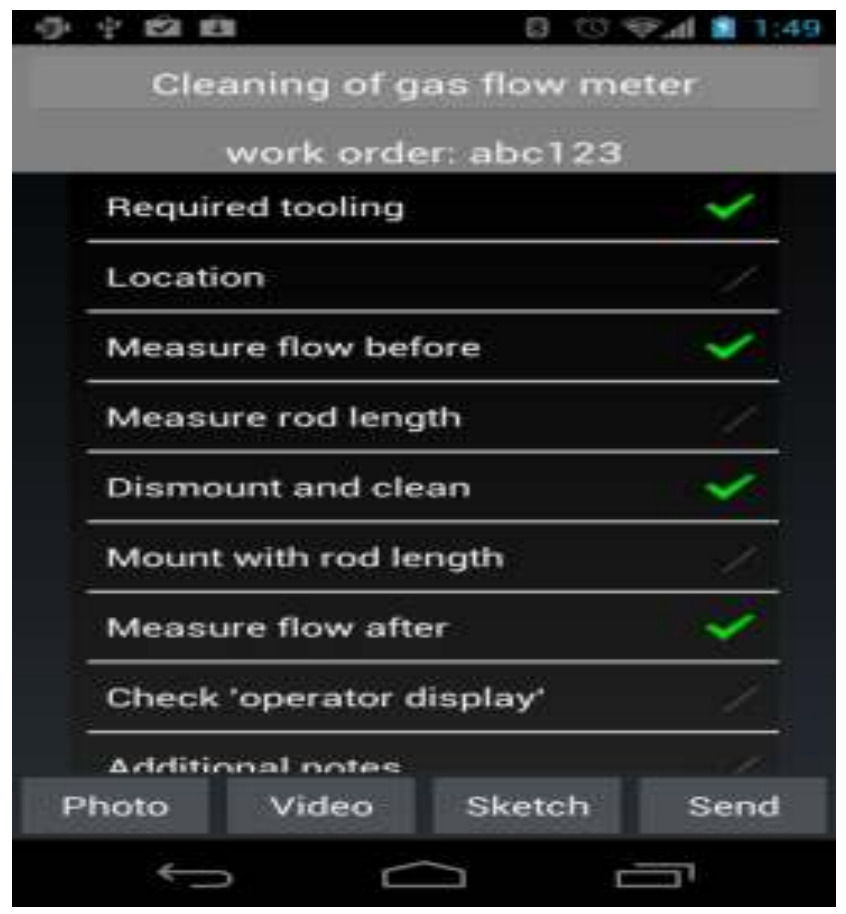

Figure 2: Screenshot of one sample work order

user interface (GUI), a business logic tier, and a data storage tier as discussed by Orfali et al. [20]. The business logic tier abstracts the interaction between the graphical user interface and the permanent / temporary data storage field. The proposed architecture was implemented using software design methodologies. For example; figure 1 below shows the interaction and messaging sequence diagram drawn to realize the annotation and AR engine modules.

As depicted in figure 2, the prototype has components that enable the service engineer to follow list of work orders for a given day as well as record media relevant information for further processing. 


\section{B. System Design Alternatives}

Software design methodologies, in general, describe a proven solution to a recurring design problem, placing particular emphasis on the context and forces surrounding the problem, and the consequences and impact of the solution. Design methodology is an interdisciplinary topic, attracting researchers from miscellaneous design disciplines such as architecture, engineering and industrial design [18].

There are many deriving reasons to use software design methodologies in developing Smartphone software:

- Patterns capture the static and dynamic structure and collaboration among key participants in software designs

- Reusability: When a problem recurs, there is no requirement to invent a new solution, as it is possible to follow the pattern and adapt it as necessary.

- Expressiveness: Design patterns provide a common vocabulary of solutions, which can be used to express larger solutions succinctly.

Model-View-Controller (MVC) is mostly used for small applications such as prototypes that are easy to manage and have simple navigation interfaces [19]. MVC is also suitable for object oriented design idioms such as Android programming. Another motivation of using MVC is to make the model independent from of the views. If the model had to notify the views of changes, it would require the reintroduction of the dependency that the software developer looks to avoid. Another design choice is the Model-View-Presenter (MVP). Unlike MVC, on the other hand, MVP is introduced for test driven development and decoupling of presenter to the model by using passive view. But it is difficult to manage for multiple views.

\section{Technologies Used}

In this project, a number of the newly emerging android Smartphone features such as AR, audio / video recording and processing, near field communication sensing and textual annotation were used.

\section{i. $\quad$ Samsung Galaxy Nexus}

A pre-study in the project suggested that the Smartphone was required to have camera, GPS, NFC, wireless local area network, zooming, and document viewer. The new Samsung Galaxy Nexus running on Android 4.0 fulfills the requirement.

\section{ii. $\quad$ Augmented Reality}

$\mathrm{AR}$ is one of the latest advancements of Human Computer Interaction (HCI) technology and context-aware computing environments that allows users to view digital information superimposed onto reality with the aid of cameras, GPS, compasses and accelerometers ([2], [3], and [4]). It combines the real and the virtual world to assist the user in performing a task in a physical environmental typesetting by sorting and seamlessly overlaying data based on geographic locations or image processing of real world objects [5].

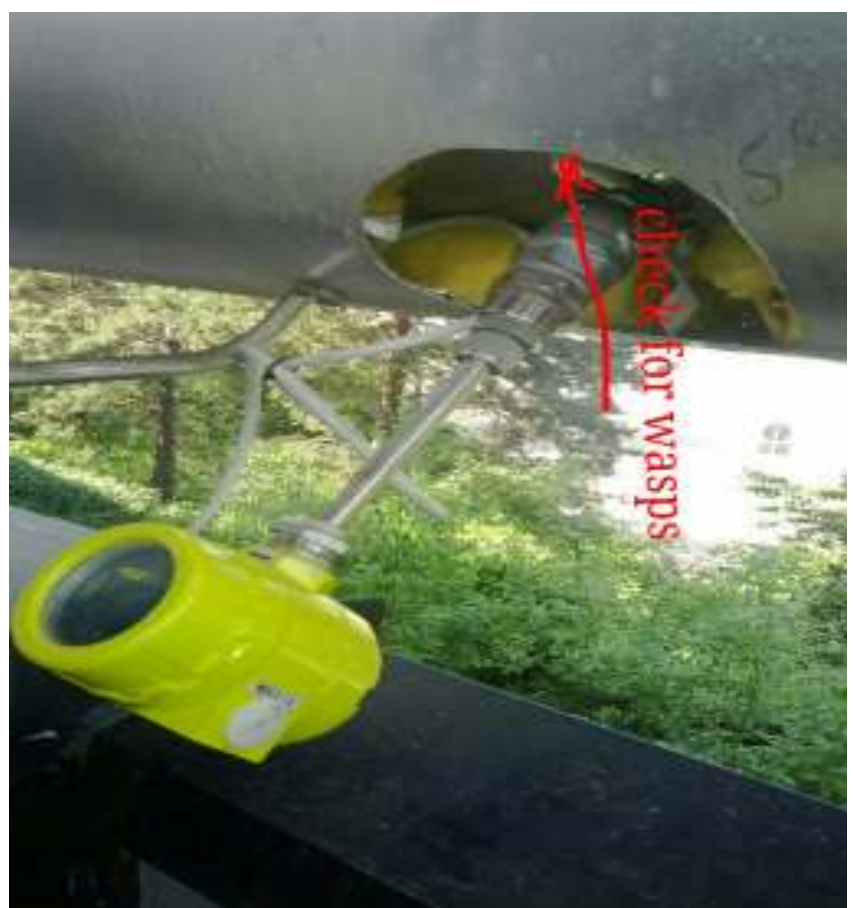

Figure 3: Text annotation on gas flow meter in the target plant site

In this project, we have used an open source $A R$ engine to superpose content over selected points of interest (POIs). The POIs were given to the engine in the form of JavaScript Object Notation (JSON) file, which stores text based association of attribute-value chain in a human-readable form [7]. The engine then fetches the value from the JSON file and superimposes the location descriptive content such as the description of a given machine over the Smartphone camera view.

\section{iii. $\quad$ Near Field Communications (NFC)}

NFC is a subset of the RFID technology, which operates in a bidirectional point-to-point short-range communication technology that allows interaction at a distance of approximately $5-10 \mathrm{~cm}$ [6]. It is an RFID deviate of $13.56 \mathrm{MHz}$ frequency communication device. NFC has become a prevalent feature in most newly emerging Smartphone devices such as the Samsung Galaxy Nexus. The NFC technology in general has two major specifications: ISO / IEC 14443 and ISO / IEC 18000-3 [7].

The objective of utilizing NFC tags in this project is to tag different machineries with unique NFC tag IDs and display relevant information pertinent to that machine as the service engineer taps his / her Smartphone over the tag.

\section{iv. Image Processing App and Text Annotation Engine}

The prototype includes text annotation and image processing engine. This part of the integral prototype was used to annotate event descriptive textual information over an image taken via the Android Smartphone. This kind of information written on a picture of machinery will be used by the service engineers to convey the behavior of the machine at a particular time and the cautions the next service engineer needs to 
consider. As depicted in figure 3, the service engineer on the prototype test leaves a message to the next engineer by taking the picture of the gas flow meter and annotating textual information over it.

\section{Prototype Implementation}

The implementation work was on instantiating the Samsung Galaxy Nexus Android hardware to record, play, superimpose and display contents as well as storing the raw data for further use. This involves implementing an appropriate data storage and retrieval technique.

The android platform provides three alternatives of versatile data persistence techniques namely shared preferences, local files, and SQLite databases [23]. Shared Preferences are effective for iterable key/value pairs, but aren't really designed for relational data. When storing the user interface (UI) state, user preferences, or application settings, SharedPreferences give a lightweight mechanism to store a known set of values. They're also meant to store a finite amount of data, not an arbitrarily large dataset [22]. For instance, an application can save the measurement of a given industrial equipment at one time in SharedPreference, but probably not a complete history of the device behavior. SQLite on the other hand is much better at relational data, since it's a relational database, but it might be an inefficient choice if the application requires retrieving one object data at a time. Typically the application needs to implement a content provider to handle data management.

A third option of data storage and retrieval in the Android platform is files [22]. Android lets programmers create and load files on the device's internal or external media.

The prototype was, therefore, designed based on a combination of the above data storage and retrieval techniques. Storing key-value measurements of work orders utilized the SharedPreference while most of the multimedia data was stored using the external and internal file storage of the Android.

\section{EXPERIMENTS AND EVALUATION}

The main goal of the experiments and prototype performance evaluation efforts were to understand how the developed prototype fits into the demands of the target field service engineers. This creates a good opportunity to test the usability of the software with the service engineers executing their real work orders live by following the prototype functionalities.

The questionnaire was prepared with the implicit intention of achieving the following:

1. Whether the functionality of the prototype system in principle can do what is needed.

2. The individual user's assessment of the extent to which the technology meets the user's high priority needs.

3. Whether the system can be used for its intended purpose.

4. To assess if the completeness with which specified users can achieve specified goals in the given scenario.

5. Whether industrial field service engineers have the readiness to adopt new technologies and solutions that come along the emerging Smartphone eco system.

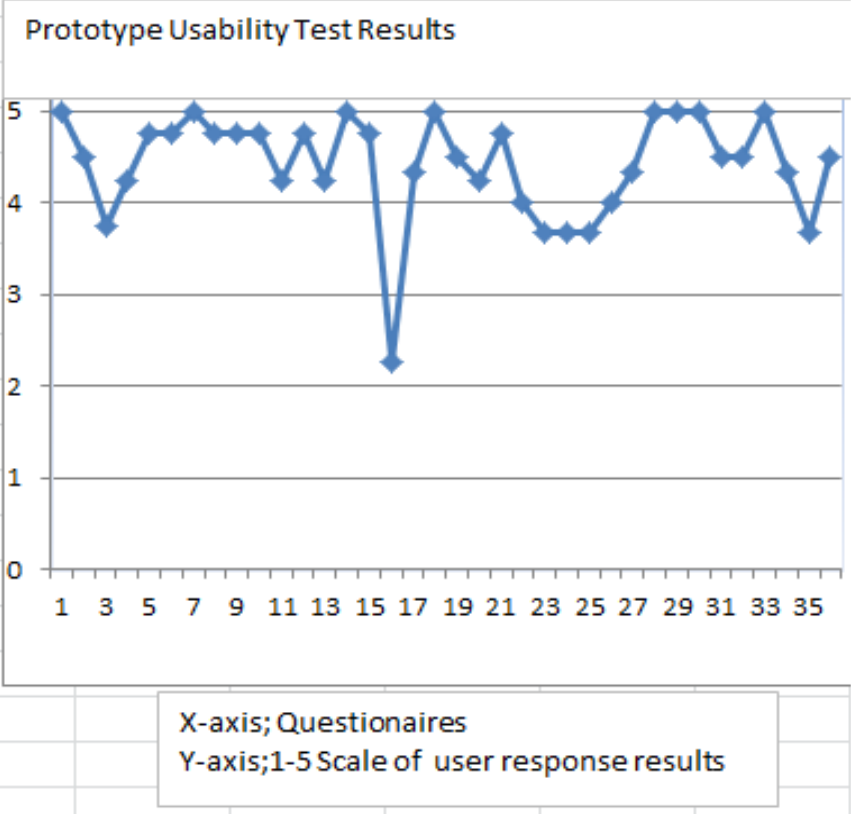

Figure 4: Quantitative analysis of the usability test.

6. How comfortable and acceptable the system is to its intended users

The average of each question is computing and depicted below in the graph. Most of the questions except for the usability of the annotation component score well above four out of five. The users were found to have relatively higher interest on AR and less on text annotations.

The interview was collected according to the following grading scale for each of the questionnaires:

5-strongly agree

4-agree

3 -neither agree nor disagree

2-disagree

1 -strongly disagree

\section{RESULTS}

After completing the development and deployment, we had a one-day experiment with ABB service engineers. The engineers range from experienced to inexperienced Smartphone users. User feedback and quotes were also collected after the field trip to the target plant where the work order executions were done live from the Smartphone. This feedback will be part of the future works in the project.

Some of the anonymous quotes about the prototype by the test service engineers include:

"That is smart doing your homework."

"It couldn't have been any easier."

"I found it quite easy. I found it smart actually."

"I hope it will come soon."

Evaluation of the questionnaires is depicted in figure 4. 


\section{A. Questionnaire Results Evaluation}

A total of four users were allowed to experiment on the prototype.

\section{i. $\quad$ Usability Experts}

Two software usability experts from ABB Corporate Research did a pre-usability test on the prototype before the actual test with the service engineers. Their feedback and usability test questionnaire results were recorded and analyzed. In general, the experts didn't have problem using the prototype but they had slight difficulty matching the workflow as programmed in the prototype and the actual work order flow. The software usability experts will not be able to ascertain if the prototype satisfies points number 4 and 5 of section IV hence a lower average score of for questionnaires 3, 15-17 and 35 .

\section{ii. $\quad$ Service Engineers}

The other test targets were the service engineers. Two service engineers were allowed to do a live test of the prototype replacing their conventional paper-pencil usage for one day. More interestingly, one of them is a regular Smartphone user while the other one rarely does. Apparently, the prototype was easy to use even for the less-experienced Smartphone user. He was quoted as saying "It couldn't have been any easier." Nonetheless, both of them experienced a slight technical flow challenge especially when trying to annotate textual descriptive information over an image captured by using the prototype, hence a lower score at questionnaires 15-17 as depicted in the graph above. A lower scale score would mean fulfilling less of point number 6 of section IV.

As depicted in figure 3, the text annotation feature enables service engineers to convey eventful messages to the next service engineer.

\section{CONCLUSIONS AND FUTURE WORK}

Today, field service engineers and trades have on-site limited information available to performing service tasks. For instance information is needed to find the right equipment onsite, to analyze the application environment, to diagnose the root-cause of the problem, and to repair the faulty equipment. Especially during site audits information must be collected to outline the current state of the installed equipment. In addition for full service or safety audits a set of tools is used to calculate and outline how the plant health and safety could be improved and guaranteed.

Mobile computing seems to hold a promising future towards implementing solutions that can alleviate the limited on-site information availability to help execute field service engineering tasks faster and more conveniently. It is even more promising with the newly emerging digital technology features available in Smartphones such as camera, video, sound recording, annotations, digital maps and AR. This project work has proved the possibility of integrating these latest technologies to develop a stable functional prototype to help field service engineers facilitate their daily work order executions such as investigating problems and troubleshooting as well as service report and documentation. The prototype developed with due consideration to functionality was found to meet the requirements of the target field service engineers which the project scenario focuses on.

An analysis of prototype usability test carried out live with the service engineers executing their daily work orders with the help of the prototype showed that the service engineers are, indeed, in need of mobile computing solutions to assist them. It indicated that solutions based on Smartphones could result in higher worker productivity, convenient information presentation, and faster and more efficient work order resolution and reporting.

\section{ACKNOWLEDGMENT}

This work was supported by ABB AB Corporate Research.

\section{REFERENCES}

[1] M. Aleksy and B. Stieger: Challenges in the Development of Mobile Applications in Industrial Field Service. Proceedings of the 12th International Conference on Network-Based Information Systems (NBiS 2009), 19.-21. August 2009, Indianapolis, USA, IEEE Computer Society, pp. 586-591.

[2] M. R. Lyu, I. King, T. T. Wong, and E. Yau: ARCADE: Augmented Reality Computing Arena for Digital Entertainment. Proceedings of the 2005 IEEE Aerospace Conference, 5.-12. March 2005, Big Sky, USA, pp. 1-9.

[3] H. Kimura, E. Tokunaga, and T. Nakajima: System Support for Mobile Augmented Reality Services. Proceedings of the ACM Symposium on Applied Computing (SAC '07), 11.-15. March 2007, Seoul, Korea, pp. 1616-1627.

[4] T. H. Höllerer and S. K. Feiner: Mobile Augmented Reality. In H. A. Karimi, \& A. Hammad (eds.): Telegeoinformatics: Location-Based Computing and Services. CRC Press, 2004, pp. 221-260.

[5] E. Dubois and L. Nigay: Augmented Reality: Which Augmentation for Which Reality? Proceedings of DARE 2000 on Designing Augmented Reality Environments, 11.-14. April 2000, Elsinore, Denmark, ACM, pp. 165-166.

[6] R. Steffen, J. Preißinger, T. Schöllermann, A. Müller, and I. Schnabel: Near Field Communication (NFC) in an Automotive Environment. Proceedings of the Second International Workshop on Near Field Communication, 20. April 2010, Monaco, pp. 15-20.

[7] Near field communication. [Online]. Available: http://www.nearfieldcommunication.org.

[8] R. Johnson, Z. Wang, C. Gagnon, and A. Stavrou: Analysis of Android Applications' Permissions. Proceedings of the 2012 IEEE Sixth International Conference on Software Security and Reliability Companion (SERE-C '12), 20.-22. June 2012, Gaithersburg, USA, IEEE Computer Society, pp. 45-46.

[9] R. Paucher and M. Turk: Location-Based Augmented Reality on Mobile Phones. Proceedings of the 2010 IEEE Computer Society Conference on Computer Vision and Pattern Recognition Workshops (CVPRW), 13.-18. June 2010, San Francisco, USA, pp. 9-16.

[10] P. D. Ritsos, D. P. Ritsos, and A. S. Gougoulis: Standards for Augmented Reality: A User Experience Perspective. International AR Standards Meeting,17.-19 February 2011, pp. $1-9$.

[11] M. Weiser: The Computer for the 21st Century. Scientific American, September 1991. 
[12] R. Ballagas, J. Borchers, M. Rohs, J. G. Sheridan, J. Foley, V. Wallace, and P. Chan: The Smartphone: A Ubiquitous Input Design. IEEE Pervasive Computing Vol. 5, No. 1, Jan. 2006, pp. 70-77.

[13] F. Michahelles, F. Thiesse, A. Schmidt, and J. R. Williams: Pervasive RFID and Near Field Communication Technology. IEEE Pervasive Computing, Vol. 6, No. 3, Jul. 2007, pp. 94-96.

[14] J. Ylinen, M. Koskela, L. Iso-Anttila, and P. Loula: Near Field Communication Network Services. Proceedings of the Third International Conference on Digital Society (ICDS '09), 1.-7. Feb 2009, IEEE Computer Society, Cancun, Mexico, pp. 89-93.

[15] D. Uckelmann, M. Harrison, and F. Michahells: Architecting the Internet of Things. Springer, 2011.

[16] J. Schiller and A. Voisard: Location-Based Services. Morgan Kaufmann Publishers, 2004.

[17] T. Zhu, C. Wang, G. Jia, and J. Huang: Toward Context-Aware Location Based Services. Proceedings of the International Conference on Electronics and Information Engineering (ICEIE 2010), 1.-3. Aug 2010, Kyoto, Japan, IEEE Computer Society, pp. V1-409-V1-413.
[18] J. Löwgren: Applying Design Methodology to Software Development. Proceedings of the 1st Conference on Designing Interactive Systems: Processes, Practices, Methods, \& Techniques (DIS '95), ACM, pp. 87-95.

[19] F. Buschmann, R. Meunier, H. Rohnert, P. Sommerlad, and M. Stal: Pattern-Oriented Software Architecture Volume 1: A System of Patterns, Wiley, 1996.

[20] R. Orfali, D. Harkey, and J. Edwards: Client / Server Survival Guide, 3rd ed. Wiley, 1999.

[21] Y. Zhang and Y. Luo: An Architecture and Implement Model for Model-View-Presenter Pattern. Proceedings of the 3rd IEEE International conference on Computer Science and Information Technology (ICCSIT), 2010, 9.-11. July 2010, Chengdu, China, pp. 532-536.

[22] V. Matos: Android Persistency: Preferences. White paper. Cleveland State University

[23] Reto Meier: Professional Android Application Development. Wiley, 2009. 